Musées, Patrimoine et Culture scientifiques et techniques

$184 \mid 2019$

juillet-août 2019

\title{
Rivière après Rivière
}

Serge Chaumier et serge.chaumier@gmail.com

\section{OpenEdition \\ Journals}

Édition électronique

URL : http://journals.openedition.org/ocim/2916

DOI : 10.4000/ocim.2916

ISSN : 2108-646X

Éditeur

OCIM

Édition imprimée

Date de publication : 1 juillet 2019

Pagination : 26-31

ISSN : 0994-1908

Référence électronique

Serge Chaumier et serge.chaumier@gmail.com, «Rivière après Rivière », La Lettre de l'OCIM [En ligne], 184 | 2019, mis en ligne le 01 juillet 2020, consulté le 24 janvier 2021. URL : http://

journals.openedition.org/ocim/2916; DOI : https://doi.org/10.4000/ocim.2916

Ce document a été généré automatiquement le 24 janvier 2021.

Tous droits réservés 


\section{Rivière après Rivière}

\section{Serge Chaumier et serge.chaumier@gmail.com}

Georges Henri Rivière, l'homme orchestre (vitrine présentée dans l'exposition Georges Henri Rivière. Voir, c'est comprendre)

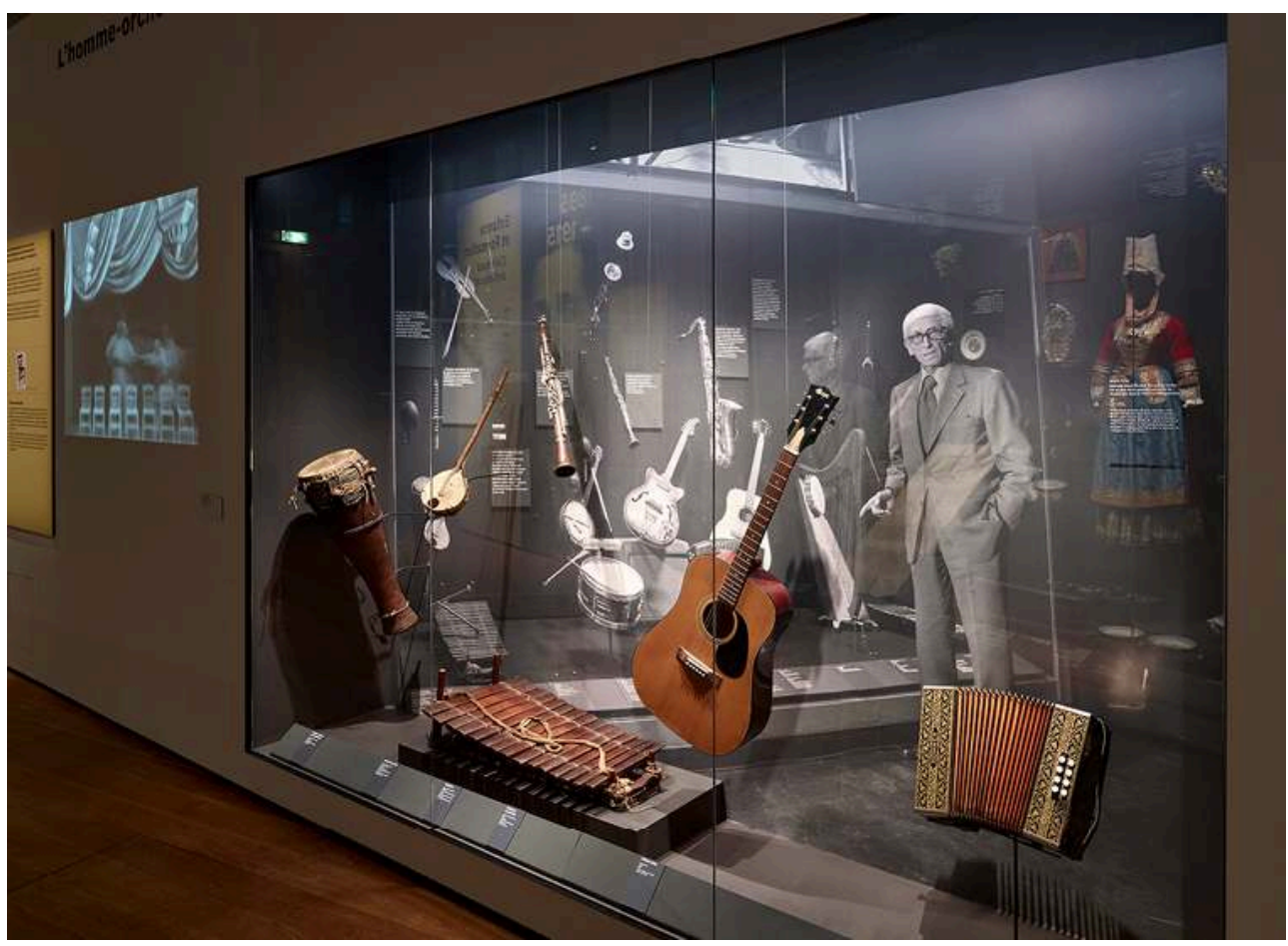

(C) Mucem/F. Deladerrire

1 Après une période dans les années 2000 où les apports de GHR semblent délaissés, voici venu le temps de la redécouverte. Les hommages surgissent et l'exposition qui lui est consacrée au Mucem enfonce le clou en dressant un portrait hagiographique du grand homme. Pourtant, peu d'écrits conduisent une lecture critique qui départagent les apports, mais aussi les limites de la muséologie selon GHR. Nous voudrions nous concentrer sur ce qui succède à Rivière en pointant, en quelques lignes, quelques 
grands axes qu'il conviendrait sans doute de nuancer et de développer dans un travail plus conséquent.

2 Si la muséologie de GHR marque l'histoire, c'est qu'elle contribue à mettre en place une méthode rigoureuse, scientifique, qui appréhende de manière systématique l'expographie, depuis les collectes, la documentation qui s'attache aux expôts, tant matériels qu'immatériels, la manière de les traiter, de les mettre en scène, de forger un discours ${ }^{1}$. Car si Rivière donne toute sa puissance à l'esthétisation pour envisager la mise en scène, n'opposant pas esthétisme et connaissance, c'est qu'il croit à la force d'imposition des images créées. Toutefois, ce serait aller un peu vite d'en conclure que « Voir, c'est comprendre », car Rivière est aussi celui qui introduit ce que l'on nomme désormais les médiations. Avec de l'écrit, mais aussi des technologies alors d'avantgarde, maquettes, audiovisuels et montages sonores si besoin. C'est le contemporain d'une sociologie de la culture qui démontre que les codes culturels sont nécessaires pour appréhender intellectuellement, mais aussi émotionnellement les contenus transmis. Il y a chez Rivière une démarche qui allie différents registres pour permettre une compréhension globale de l'Homme en son milieu.

3 Si GHR a une influence considérable par son enseignement et par sa position institutionnelle au sein de l'Icom sur la muséologie mondiale, on ne devrait pas oublier pour autant d'autres approches tout à fait complémentaires voire redondantes, portant les mêmes objectifs. C'est le cas de Jean Gabus, muséologue suisse qui va également apporter une forte modernisation aux antiques musées d'ethnographie ${ }^{2}$. GHR met en œuvre le musée laboratoire, concept défendu par Paul Rivet, réunissant des équipes pluridisciplinaires dans des missions scientifiques qui n'oublient pas de relever l'art comme une des données de la vie sociale. Pourtant, si le musée national des arts et traditions populaires rentre assez vite dans une crise durable, quelques temps après son inauguration, il reflète avec un temps d'avance ce que les musées régionaux vont connaitre par la suite.

Certes, les années 1970 et 1980 voient se multiplier les désirs de musées, et de nombreuses initiatives surgissent sur le territoire pour donner naissance à des associations générant des musées bientôt professionnalisés. Malgré tout, ils incarnent moins la muséographie de Rivière qu'un mixte avec ce qui apparaît déjà comme un renouveau, à savoir des musées de société pensés à partir de ce que l'on appelle au détour des années 1980 "la nouvelle muséologie $»^{3}$. Celle-ci est souvent associée à l'écomuséologie, car de nombreux acteurs appartiennent aux deux mouvements, cependant on peut les distinguer par les valeurs qui ne sont pas exactement les mêmes. Sans revenir ici sur l'écomuséologie dont la paternité nous semble plus revenir sur le fond à Hugues de Varine (par le souci donné à la participation des communautés locales et à leur priorité en dernière instance), qu'à GHR dont on sait l'attachement aux collections et aux objets, mais aussi à la primauté du scientifique. Questions que nous avons traitées ailleurs ${ }^{4}$.

\section{La place du public}

5 La nouvelle muséologie conserve de Rivière la priorité donnée au public. Le musée n'a pas de sens sans ses destinataires. Si ce point est commun, il connaît dans l'époque post-Rivière une augmentation de son impact. La sociologie, comme la réflexion sur les politiques culturelles, conduisent dans les années 1970 et 1980 à pousser en avant 
l'action culturelle, l'animation, puis la médiation non pas comme un pis-aller ou une extension des fonctions de l'institution (comme il est encore souvent coutume dans les musées d'arts), mais comme le centre de gravité même du projet des musées qu'éliane Barroso et Émilia Vaillant proposent alors d'appeler "de société $»^{5}$. Nombre de militants de la cause des publics vont porter l'argumentaire, et souvent le rapport de force pour se faire entendre, et parvenir à son inscription dans la loi musée de France en 2002. Si la question était bien présente, elle ne semblait pas aussi centrale dans la muséologie de Rivière. Avec le temps, le rapport devient de moins en moins descendant, de l'expert au profane, et de plus en plus co-construit. À un Homme universel abstrait dont on entend rendre compte, notamment au travers de ses fonctions et de son statut dans une société, succède la présentation d'individus singuliers au travers de témoignages et de paroles subjectives, davantage inscrite dans le courant d'une histoire des mentalités et de la micro-histoire, productrice de la nouvelle histoire.

À Neuchâtel, dans le Musée dynamique, de la galerie en direction du sud-est, Jean Gabus proposait de juin à décembre 1963, l'exposition La main de l'Homme.

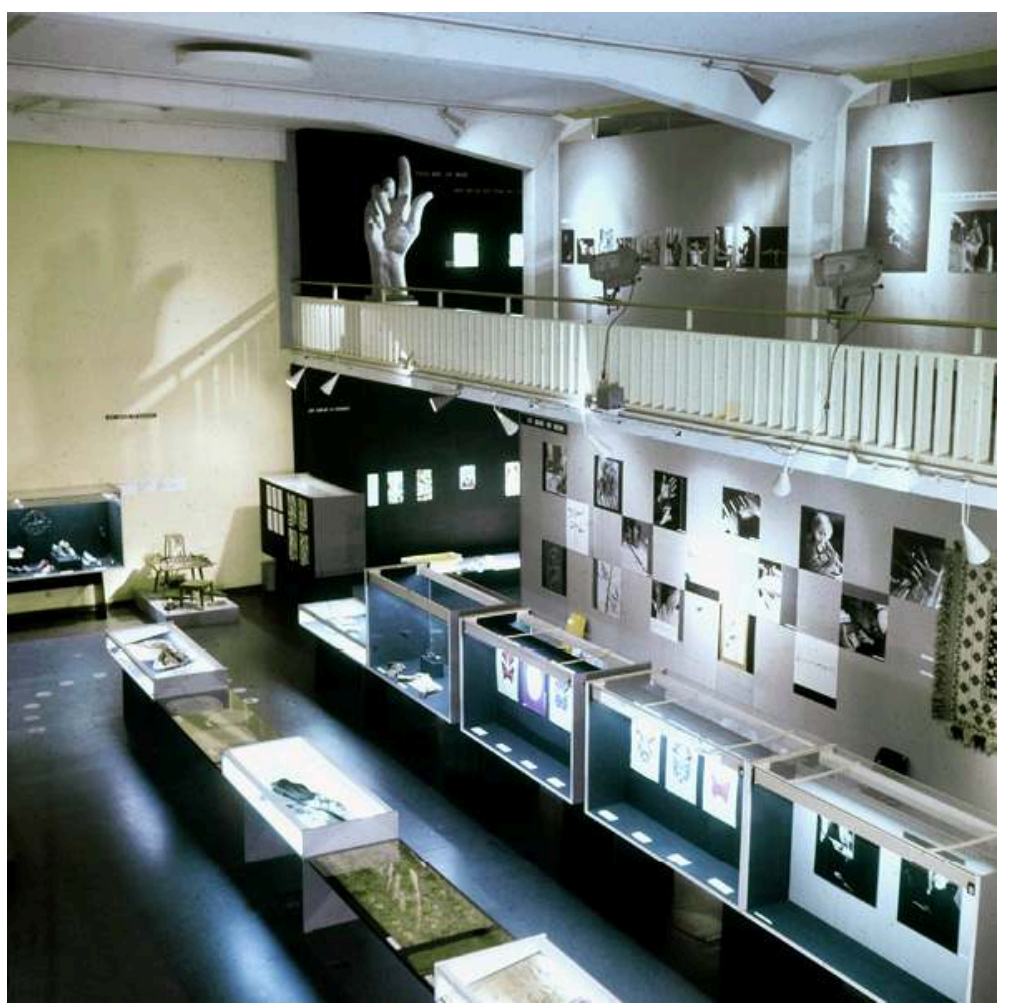

(c) Musée d'Ethnographie de Neuchâtel/Walter Hugentobler

\section{L'émergence du muséographe}

6 Le programme muséographique établi par Rivière apparaît comme l'ancêtre de ce que l'on désigne désormais comme une " muséographie de discours ", car il ne s'agit pas seulement de montrer, mais de faire parler les expôts pour signifier quelque-chose, chez Rivière la compréhension de l'Homme dans son environnement. C'est là où la muséologie des années 1980 dépasse cette approche, pour proposer autre chose. 
Toutefois, il serait abusif d'assimiler le programme tel qu'il est conçu dans les années 1960 et celui produit aujourd'hui par un muséographe. Du reste, le nom lui-même exprime cette évolution, chez Rivière le muséographe assume tout, de la collecte à la préservation, du discours à la composition des mobiliers d'exposition en passant par les outils d'informations. C'est l'homme-orchestre qui rassemble en une seule personne ce qui va devenir divers corps de métiers par la suite : conservateur, mais aussi préventeur, régisseur, muséographe et scénographe ${ }^{6}$. Désormais seuls quelques musées d'art confondent encore ces deux derniers métiers, comme paradoxalement encore bloqués à l'époque de Rivière! Les musées d'ethnographie, à l'instar des musées de science, font la différence, parce que ces deux métiers ont justement connu des évolutions notoires. Et c'est le dépassement de la muséologie de Rivière qui les rend possible.

\section{Le développement de la scénographie}

7 Soulignons donc le troisième point qui nous paraît être caractéristique des apports de la nouvelle muséologie, à savoir l'émergence de l'expographie, que l'on va bientôt nommer scénographie. Or, si on peut voir rétrospectivement une scénographie particulière chez GHR, il n'empêche qu'elle ne recouvre pas alors la même démarche. Elle est d'abord l'expression de ce que le chercheur doit traduire du terrain où il a produit sa collecte. Il s'agit de mettre en scène avec la plus grande exactitude une réalité dont on entend témoigner. Avec Jean-Pierre Laurent, au musée dauphinois, s'invente une autre approche. Celle-ci fait le choix de la métaphore, du symbolique, voire du conceptuel pour apporter un véritable niveau de lecture supplémentaire. Dès lors un métier s'invente qui va se professionnaliser et connaître le succès que l'on constate depuis 30 ans maintenant. L'association des scénographes en témoigne par le nombre d'agences qui ont vu le jour. Rappelons que Jean Gabus conçoit une vitrine qu'il espère universelle, idéale, utilisable pour toutes les expositions temporaires! Nous sommes bien loin à l'époque de la volonté d'affirmer une créativité singulière pour chaque sujet.

Il nous semble que ces trois ordres, primauté du public, muséographie et scénographie, sont les trois facteurs pour comprendre en quoi la muséologie de Rivière va connaître des dépassements.

Dans l'unité écologique "Le buron de l'Aubrac ", le diorama se réinvente dans la mesure où il doit obéir le plus fidèlement que possible à une réalité dont l'ethnographe veut témoigner. Les relevés sont méthodiques et la composition doit faire percevoir une photographie matérielle d'un instant donné. Dans la composition « Du berceau à la tombe ", et même si les objets proviennent de plusieurs provenances, offrant une perception géographique hétéroclite, il s'agit de faire comprendre l'universalité de rites ponctuant la vie humaine des sociétés en question. C'est un message qui entend rendre compte d'une analyse scientifique objective. Y compris dans les présentations sérielles, les objets mis en série cherchent à témoigner de la diversité des formes dans l'unité des productions. Bref, un objet tend à signifier avec force objectivité un état donné d'une société humaine. C'est bien là que le bouleversement va avoir lieu. 
Objets prétextes, objets manipulés, une exposition organisée sous la direction de Jacques Hainard et présentée au musée d'Ethnographie de Neuchâtel de juin à décembre 1984.

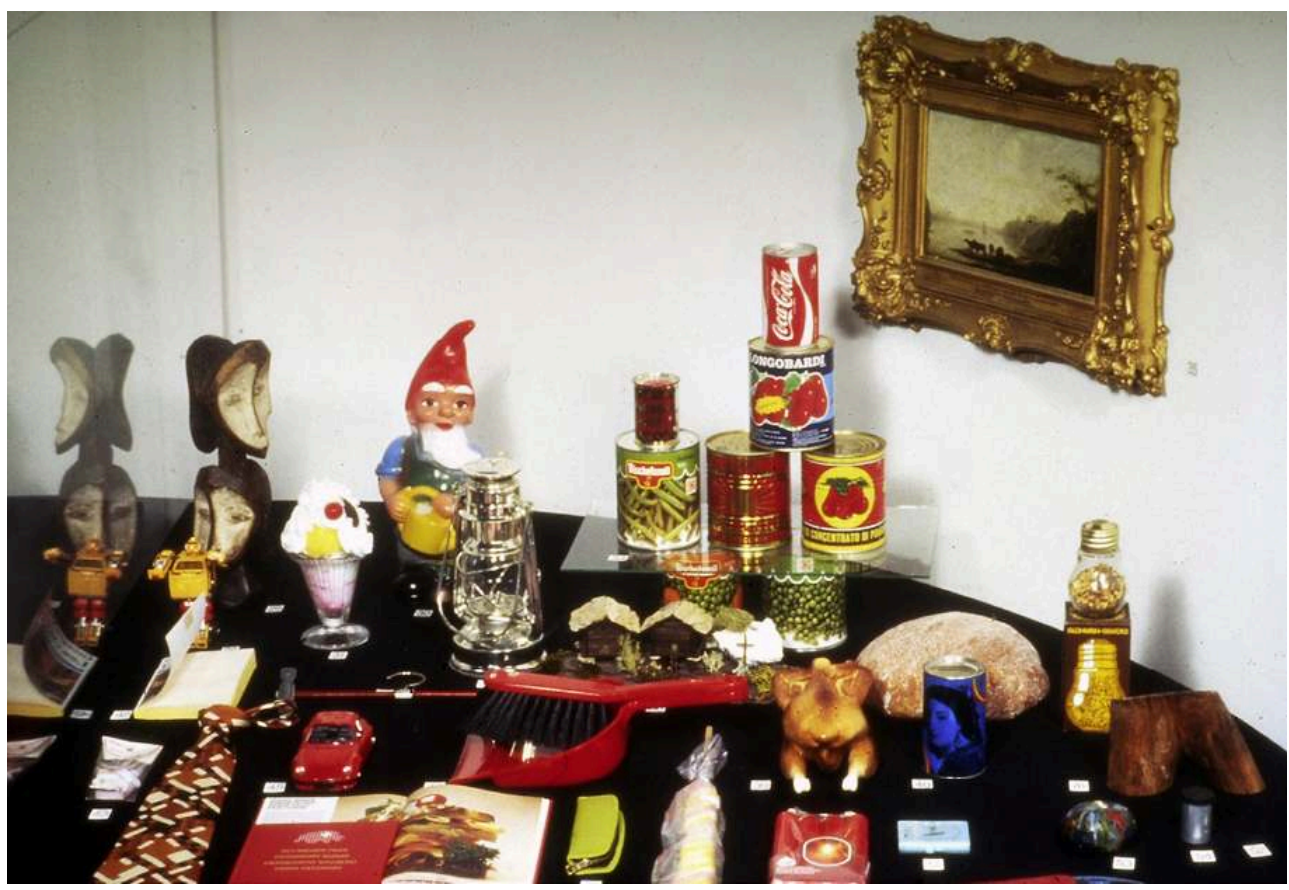

(c) Musée d'Ethnographie de Neuchâtel/Alain Germond

\section{Au-delà de l'objet}

10 Après les années 1980 , le changement de générations rend de plus en plus difficile la compréhension immédiate des expôts par leur dialogue et leur composition. La référence à la société rurale se perd avec les codes de lecture. Mais plus encore, des muséologues découvrent que "l'objet n'est la vérité de rien du tout ", selon le bon mot de Jacques Hainard ${ }^{7}$. Tout discours est socialement et historiquement situé, quand bien même il s'incarnerait par des objets. Il ne suffit pas de montrer pour signifier, et même accompagné de médiations, un objet n'a que l'apparence de son objectivité. Chaque expôt est un sémiophore qui porte potentiellement une infinité de signifiants, selon le contexte et les autres expôts qui l'entourent. Dans cette veine, Martin Schärer va proposer un exercice célèbre à partir d'une soupière, pour en dévoiler les multiples registres d'interprétation ${ }^{8}$. Il appartient donc au muséographe de décider du discours qu'il tient, plutôt que de faire croire que la mise en scène des objets conduit à dire le vrai. C'est ce que va résumer la muséologie de la rupture, selon l'école neuchâteloise.

11 À partir de ce courant, la muséologie objectiviste, pour ne pas la qualifier de positiviste, de Rivière ne peut plus être univoque, elle ouvre le champ des possibles. La muséologie de l'interprétation qui se répand dans les années 1990 depuis le Québec, à partir de la lecture de Tilden, le signifie autrement ${ }^{9}$. Dès lors qu'il y a de multiples interprétations, il y a des choix d'auteur à faire de la part de l'équipe de conception muséographique. Cette œuvre de l'esprit se prolonge par la volonté de l'exprimer de manière singulière, discours incarné et prolongé par les apports de l'équipe scénographique. Celle-ci s'enrichit de compétences métiers : au scénographe, il faut ajouter graphiste, manipeur, réalisation audiovisuel, multimédia, acousticien, voire artiste en tous genres. 
Bref, l'exposition devient d'autant plus unique qu'elle est par elle-même une œuvre de création, qu'il est même possible de la signer comme œuvre en soi. En général, c'est une œuvre composite, élaborée par un collectif, mais elle peut aussi, plus rarement, être la production d'un artiste qui en assume les différentes facettes.

\section{L'émergence d'un nouveau paradigme}

12 Ce qui précède conduit à revendiquer pleinement l'exposition comme une œuvre unique, mais aussi une œuvre finie par la réception qui en est opérée, puisqu'elle fait de plus en plus la part belle aux visiteurs. Ce sont eux qui la finissent par leurs usages pluriels. Les registres d'interprétation proposés sont nourris et s'enrichissent de leur réception. Les visiteurs tiennent un rôle, qui va jusqu'à l'implication dans la co-écriture et la finalisation de l'exposition ${ }^{10}$. Dans la mesure où le concepteur leur donne de plus en plus de place dans l'appropriation, l'exposition devient pleinement expérientielle. Terme que l'on voit se diffuser à partir des années 2000, et qui signifie suffisamment que l'exposition se complexifie par son usage. Les registres mentionnés brièvement sont de différentes natures selon les cas et une pluralité peut être recensée. Ce qui importe, c'est de noter ce dépassement de la muséologie de Rivière, par le discours interprétatif, par l'explosion des formes, par le rôle tenu par les visiteurs.

Ces trois ordres formant une triangulation qui a des effets induits. On peut citer par exemple une certaine désacralisation de l'objet. Le développement de l'interactivité en est révélateur, Jean-Claude Duclos rappelle les tensions entre Rivière et Jean-Pierre Laurent qui propose de toucher certains objets. Un autre signe est le recours aux nombreux artefacts, comme dans le courant de l'interprétation qui mêle les copies aux objets de collection, sans que cela ne choque tant c'est le discours tenu qui finalement prend le pas. Dans l'exposition d'auteur, on ne vient plus voir une collection mais une thèse soutenue (voir les expositions aussi différentes que celles produites par le muséum ou le musée d'ethnographie à Neuchâtel, ou encore La Mélancolie de Jean Clair ${ }^{11}$. L'affirmation de vouloir s'adresser à l'ensemble de la personne, son intellect, mais aussi ses sens, ses émotions, son imaginaire, sa créativité... sont autant de facteurs qui conduisent au passage d'une forme de valorisation discrète, pour ne pas dire de décoration, à une véritable scénographie. Au sens fort du terme celle-ci porte un autre niveau d'appréhension, voire un métadiscours. Elle l'emporte alors par son registre expérientiel, au grand dam des tenants de l'approche classique qui entendent que la scénographie se fasse discrète et humble pour mettre en valeur les collections, ne comprenant pas que c'est le registre même de la proposition qui est devenu d'une toute autre nature ${ }^{12}$. 
L'exposition Gens de là-haut muséographiée par Jacques Laurent, et inaugurée en 1977 au Musée dauphinois (photographie prise en 1998 avant le démontage de l'exposition).

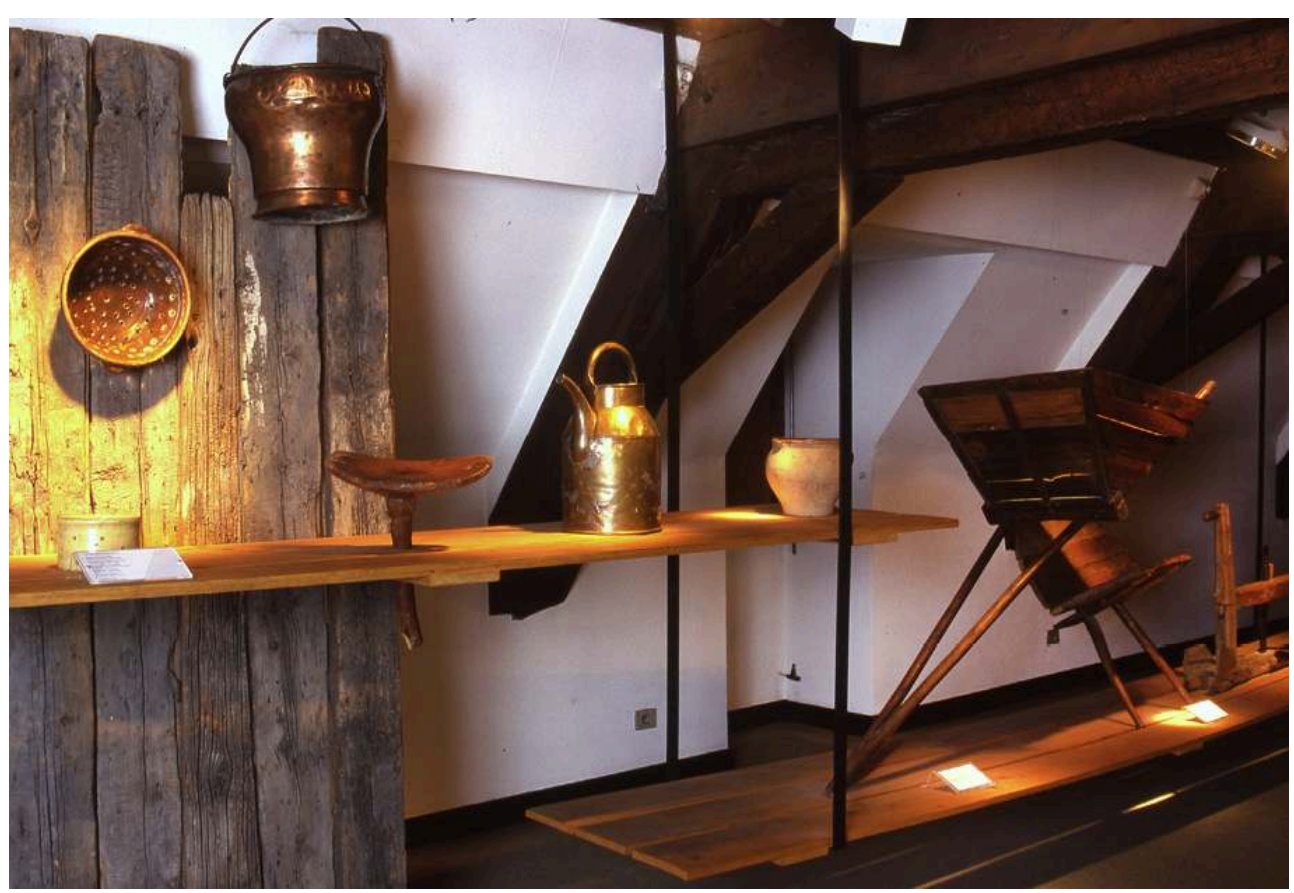

(c) Musée dauphinois - Département de l'Isère/Yves Bobin

\section{NOTES}

1. Collectif, La Muséologie selon GHR, Dunod, 1989.

2. Knodel, B. Le Musée Réinventé. MEN, Neuchâtel, 2018.

3. Collectif sous la direction d'André Desvallées, Vagues. Une anthologie de la nouvelle muséologie, MNES/ W, deux volumes, 1992.

4. Voir Des musées en quête d'identité. Écomusée / Technomusée, L'Harmattan, 2003.

5. Éliane Barroso et Émilia Vaillant, coordination, Musées et sociétés, Actes du colloque national, Mulhouse Ungersheim, DMF, Ministère de la Culture, 1993.

6. Voir Projet d'exposition. Guide des bonnes pratiques, téléchargeable sur le site de l'association des scénographes : www.scenographes.fr/scenographes.fr/documents/guideexpo_nogloss.pdf

7. GHK, 100 ans d'ethnographie sur la montagne Saint-Nicolas. MEN, 2004.

8. Schärer, M.-R. La relation Homme-objet exposée : théorie et pratique d'une expérience muséologique, Publics et Musées, n¹5, PUL, 1999.

9. Tilden, F. Interpreting our heritage. Third Edition, 1957.

10. Duclos, J.-C. La co-écriture au musée, in Chevalier, D. (dir.) La fabrique des musées de sciences et sociétés. La Documentation française, 2011.

11. Voir notre Traité d'expologie. Les écritures de l'exposition, La Documentation française, 2012.

12. GHK, 100 ans d'ethnographie sur la montagne Saint-Nicolas. MEN, 2004. 


\section{RÉSUMÉS}

Cet article est extrait de dossier "L'exposition ethnographique selon Georges Henri Rivière ». Ce dossier est l'occasion de reparler d'une question essentielle : en quoi les multiples expositions qu'a conçues et coordonnées Georges Henri Rivière ont-elles renouvelé le mode de collecte et d'exhibition des collections ethnographiques? Au point de faire de la muséologie selon GHR un tournant dans l'écriture du discours expographique et une référence connue dans le monde entier. Il s'agit de rappeler, de façon claire et concise, quels ont été les principes clefs de son inventivité et de sa créativité expographique à travers quelques exemples. Marie-Charlotte Calafat et Germain Viatte reviennent sur les restitutions fidèles réalisées pour l'exposition du Mucem. Jean-Claude Duclos évoque l'exposition initiale, fidèle à l'enseignement de GHR, qu'il a conçue en Camargue. Daniel Jacobi prend exemple du musée du vin de Bourgogne à Beaune pour souligner les qualités esthétiques de cette muséologie. Enfin, Serge Chaumier replace la période Rivière dans l'évolution des expositions dans les musées dits de société.

\section{INDEX}

Mots-clés : Georges Henri Rivière, muséographie, collections

\section{AUTEURS}

\section{SERGE CHAUMIER}

Responsable du master Muséographie, expographie, université d'Artois 